\title{
A Light and Electron Microscope Study on the Golgi Bodies in Neurones of a Pelecypod, Meretrix meretrix lusoria (Gemelin)
}

\author{
Jin-ichi Kitada \\ Department of Biology, University of Osaka Prefecture, Sakai, Japan
}

Received February 14, 1958

Though an extensive study has been made on the Golgi apparatus with the aid of a light microscope, there is considerable controversy in respect to its structural detail with divergent claims. Recently, thanks to the helpful use of the electron microscope in this field of research, a remarkable agreement by several investigators has been obtained on the fine structure of the Golgi apparatus. They have similarly described the smooth membranes, small granules or vesicles, and large vacuoles as structural elements of the Golgi apparatus in various types of cells of vertebrates (Dalton and Felix 1954, Sjöstrand and Hanzon 1954, Palay and Palade 1955, Burgos and Fawcett 1955, Watanabe, Takamatsu and Osako 1956). Further, it has been revealed that certain cell types of invertebrates show the Golgi apparatus which is basically similar in pattern to that of vertebrate cells (Gatenby, Dalton and Felix 1955, Dalton and Felix 1956, Beams, Tahmisian, Devine and Anderson 1956).

It has often been stated that the Golgi apparatus in the neurones of invertebrates appears as a diffuse complex of dots, rods, or crescents, i.e., discrete bodies which are generally called dictyosomes. It is an interesting subject to inquire with the phase optics and the electron microscope into the structural difference of the dictyosome occurring in invertebrates neurones by way of comparison with the Golgi complex of vertebrates. Along this line of investigation, the neurones of a pelecypod, Meretrix meretrix lusoria, were chosen as material for the present study, since this bivalve is easily obtainable in winter. Prior to the completion of the present report, a paper by Lacy and Rogers (1955) has appeared concerning the cytoplasmic inclusions of the neurones of Patella vulgata which were studied by light and electron microscopes. The results of the present study are to be described in comparison of those of these later authors.

\section{Material and method}

Large- or medium-sized specimens of the pelecypod, Meretrix meretrix lusoria (Gemelin), were used as the material. The cerebral and visceral ganglia were removed by cutting their anterior and posterior adductors. 
For observation of the neurones in living condition, a drop of either the body fluid of the animal, or 0.75 per cent solution of sodium chloride, or Baker's solution placed on a slide was used in the examination of fresh ganglia. After a cover-slip was placed on the material thus mounted, gentle pressure was applied on the slip. The excess of fluid was removed with blotting paper, and the coverslip was sealed with melted paraffin.

The following Tiyoda's phase optics were used for observation: DM (dark contrast medium), DL (dark contrast low), B-(bright contrast minus), and NDM (neo dark contrast medium) oil immersion objectives $(\times 90)$.

Some of the ganglia were supravitally stained with neutral red which was dissolved to a concentration of $1: 10,000$ in a 0.75 per cent solution of sodium chloride. For the sake of comparison, some material was fixed with Champy's fluid for 20 hours and then preserved in 1 per cent osmic acid at $37^{\circ} \mathrm{C}$. for 6 days, according to Kolatchev.

For electron microscopy, the neurones were fixed for an hour in 1 per cent veronal-acetate-buffered osmium $(\mathrm{pH}: 7.3)$ at $10^{\circ} \mathrm{C}$., and then washed for a minute in distilled water. The tissue was dehydrated through ascending series of alcohol and embedded in a mixture of 8 parts of normal butyl methacrylate and 2 parts of methyl methacrylate. Sections made with glass knives on a Hitachi UM-1 type ultra-microtome were examined by means of a Hitachi HU-9 type electron microscope.

\section{Observations}

\section{Observations by light microscopy}

In the preparations after Kolachev, small black bodies impregnated with osmium tetroxide are observed in the cytoplasm around the nucleus and in the axon-hillock of the neurone (Fig. 1). They vary in size assuming the form of a dot, rod or crescent. They are closely similar in ap. pearance to the dictyosomes which have been

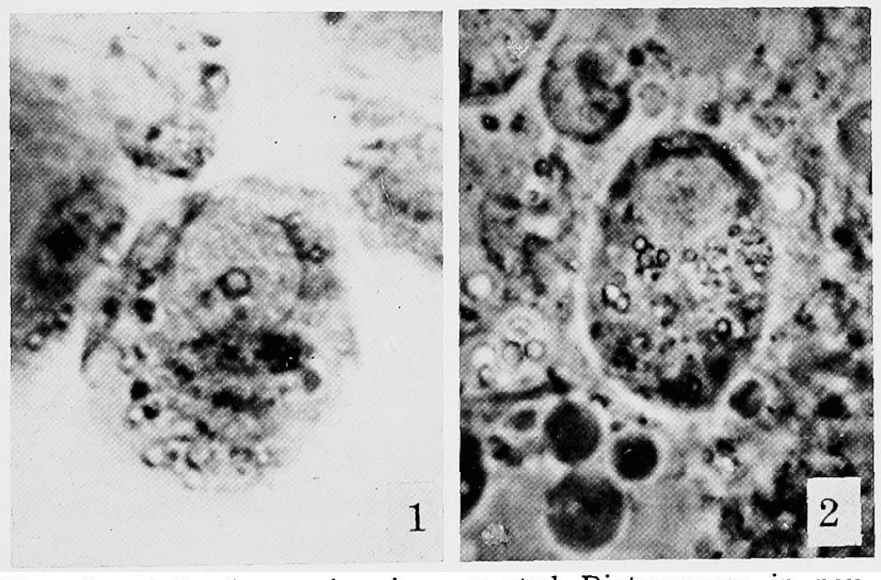

Figs. 1 and 2. 1, osmium-impregnated Dictyosomes in neurones, Osmium-impregnation after Kolatchev. $\times 2000.2$, golgi bodies in living neurones mounted in body fluid, phase contrast, $\times 2000$. described by several authors in neurones and in germ cells of certain invertebrates. They are independent in occurrence from one another in the present material, which 
has been treated with osmium tetroxide for 6 days.

As revealed by the phase contrast microscope, the fresh neurones show small round bodies which are closely similar both in size and in distribution to the black bodies observable in Kolatchev's preparations (Fig. 2). The NDM oil immersion objective was useful in observation of their detailed structure. Careful study reveals them to have a binary nature consisting of an inner part which appears bright, and an outer ring- or crescent-shaped rim which is dark. Each body is thus made up of an inner archoplasm and an outer dictyosome which form its medulla and cortex, respectively. The bodies in question are $0.7-1.0$ micron in dimension. In addition to
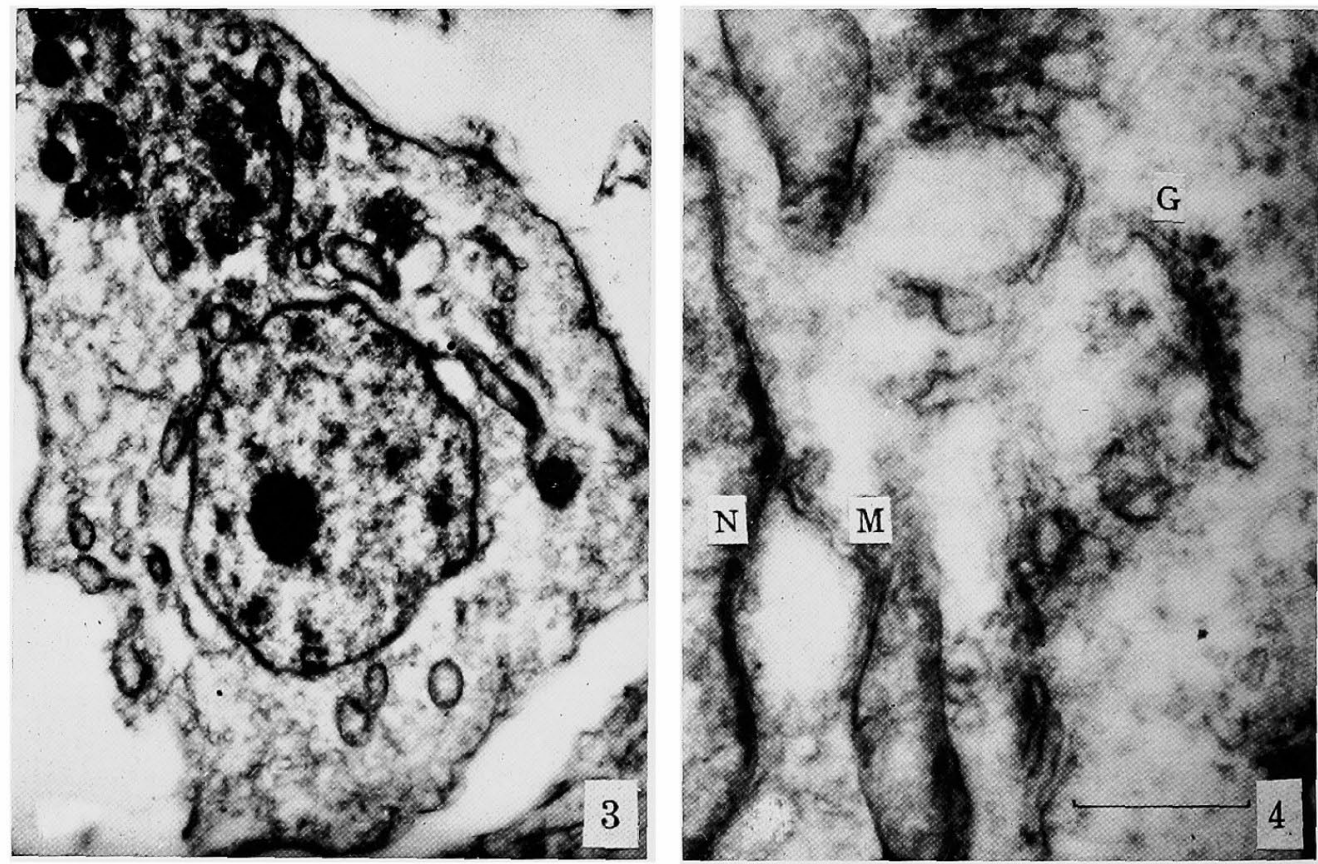

Figs. 3 and 4. 3, an electron-micrograph of a neurone. $\times 10,000.4$, a portion, upper right to the nucelus, of the same neurone as in Fig. 3, showing nuclear membrane (N) mitochondria (M) and Golgi complex (G). ×40,000.

these bodies, there are smaller granules which are dark and apparently homogeneous in nature.

The supravital staining of the neurones with neutral red indicates that these latter smaller granules are stained uniformly with the dye. Based on this staining reaction, they are considered probably to be lipochondria. In contrast to these smaller granules, the binary bodies show a differential reaction to the stain: the outer dictyosome is stained, though not intensely, with neutral red, while the inner archoplasm shows no reaction to the stain.

II. Observations by electron microscopy

Figure 3 presents an electron photomicrograph of a neurone under a low magnification. In the cytoplasm around the nucleus there are certain struc- 
tures which are irregular in form, electron-opaque and distinguishable from both the mitochondria and the endoplasmic reticulum. Under high magnifications, however, the structure is found to consist basically of lamellae and vacuoles (Figs. 4-6). The lamellae are made up of several parallel layers of double membranes, the distance between every two membranes being about $300 \AA$ А.

Most vacuoles are $0.5-0.7$ micron in diameter. In a few cases, there occur stacks of double membranes without vacuoles. However, a great majority of the double membranes show a swelling at their end, forming a vesicle or a vacuole. Most of the double membranes are slightly curved
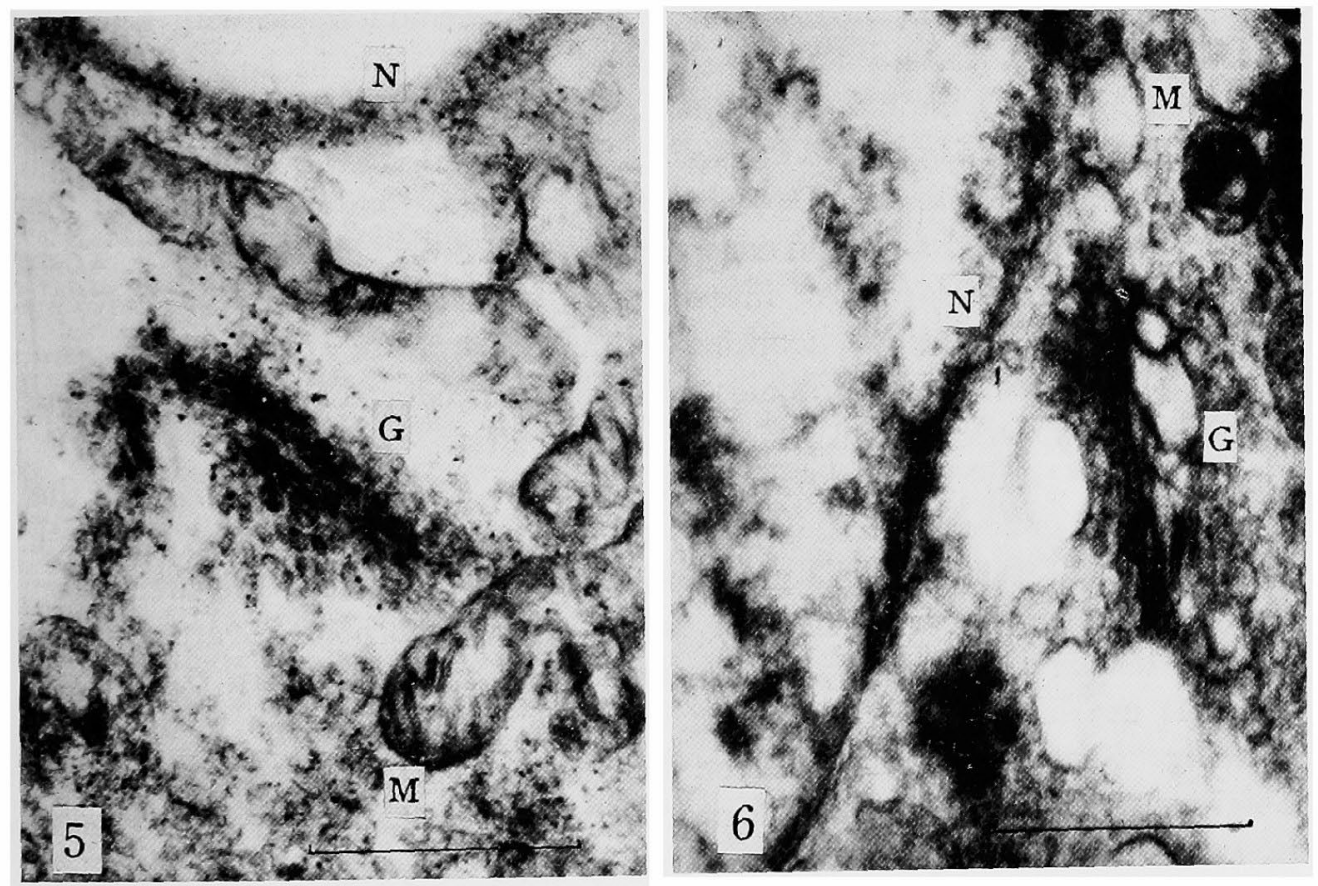

Figs. 5 and 6. A juxta-nuclear portion of a neurone from the other preparations $\times 50,000$.

in different degrees. There are instances in which a number of granules or microvesicles being in dimension about $200 \AA$ are aggregated in the neighborhood of a stack of double membranes. In all probability the components, such as double membranes, vacuoles and granules, are the elements which form the Golgi complex. Though the data now at hand are not yet satisfactory, the supposition may be allowed that the stack of paired membranes and the aggregation consisting of vacuoles and microvesicles may correspond to the dictyosomes and the archoplasm, respectively.

In addition to the above components, electron-dense spheroids without definite internal structure are observed in the cytoplasm, especially in the 
axon hillock (Fig. 3). They are strongly impregnated with osmium tetroxide, and therefore they seem to be lipoidal granules or lipochondria.

The mitochondria more or less clearly show cristae mitochondriales which run generally oblique to the surface. The outer membranes are much thinner than the inner membranes, their two opaque layers being only indistinctly indicated in places.

\section{Discussion}

Most previous authors have reached the conclusion that the small bodies occurring in the living neurones of invertebrates correspond to those composed of a dictyosome and an archoplasm which are demonstrable with the classical technique (Moussa 1950 in Limnaea; Kitada 1954 in land snails and a Prosobranch; Malhotra 1955 in Locusta and a water bug; Gresson, Threadgold and Stinson 1956 in Helix, Locusta and Lumbricus). Although there are minor differences of opinion among them, they all agree that the bodies have a duplex structure consisting of a medulla and a cortex. There is, however, another group of investigators, such as Thomas (1947), Roque (1954), Shafiq $(1953,1954)$, working on the neurones of Helix and Locusta, who emphasize that the dictyosomes are artifacts produced by Golgi technique on or in the spherical lipid bodies or lipochondria.

It has been evidently shown in the present study with Meretrix that the Golgi elements really exist in living cells, and that they consist of a medulla and a cortex. Namely, the osmiophobic medulla represents the archoplasm and the osmiophilic cortex the dictyosome. The lipochondria are separate entities, devoid of internal structure and stained uniformly with neutral red.

The use of the electron microscope has contributed much to understanding of the relationship between the dictyosomes of invertebrates and the Golgi complex of vertebrates. Beams, Sedar and Evans (1953) were the first to use the electron microscope to study the dictyosomes in invertebrate neurones in a grasshopper, Melanopus differentialis. In that study the dictyosomes were demonstrated merely as bodies relatively opaque to electrons, displaying no detailed structure. Later, Robertis and Benett (1955) have described the Golgi bodies as dense masses of double membranes with vesicles in nerve cells of the earthworm. A similar structure without large vacuoles has been described by Dalton and Felix (1956) in spermatids of Lumbricus and Helix. On the other hand, the Golgi bodies consisting of paired membranes, small granules and vacuoles have been observed in neurones of Patella vulgata by Lacy and Rogers (1955), and in spermatocytes of a cricket by Beams et al (1956). Lacy and Rogers (1955) are of opinion that the dictyosomes of the neurones may probably correspond to the paired membranes with enclosed dense substance, and the archoplasm to the small granules or vesicles. 
The results to the present study have led to the view that the Golgi bodies of the Meretrix neurones consist of three elements, similar in nature to those forming the Golgi complex of vertebrates, although they sometimes fail to accompany vacuoles. It has been shown that as in Patella the double membranes and the aggregation of vacuoles and vesicles may correspond to the dictyosome and archoplasm respectively, which correspondence is to be demonstrated by the osmium-impregnation technique.

\section{Summary}

The Golgi bodies in the neurones of the cerebral and visceral ganglia of a pelecypod, Meretrix meretrix lusoria (Gemelin), were studied in the living condition with the phase optics and in ultra-thin sections by electron microscopy, by way of comparison with those demonstrated by the classical method.

In Kolatchev preparations, the Golgi bodies appear as separated bodies in the form of a dot, rod or crescent. They correspond to the dictyosomes described by previous workers. They are particularly dense in occurrence in the cytoplasm around the nucleus and in the axon-hillock.

Observations by phase contrast microscopy reveal that the Golgi bodies in the living neurones appear as round bodies, each having a dark ring- or crescent-shaped rim and a light center. They show a structure consisting of a cortex and a medulla which correspond to a dictyosome and an archoplasm, respectively.

The dictyosomal portion of the Golgi bodies is stained with neutral red with some difficulty, while the archoplasm is inactive to the stain. The cytoplasm contains small granules or lipochondria which are distinctly and homogeneously stained with neutral red.

Each of the Golgi bodies when examined under the electron microscope is found to be a complex consisting of paired membranes, vacuoles and granules or microvesicles. It is probable that the dictyosome is composed of a stack of paired membranes and that the archoplasm is an aggregation of vacuoles and microvesicles.

\section{Acknowledgement}

The author wishes to express his sincere gratitude to Professor Shunzo Takagi, University of Osaka Prefecture for his valuable suggestions and for the improvement of the manuscript. The author is also grateful to Professor Sajiro Makino for his encouragement and reading through the manuscript preparatory to publication. 


\section{Literature}

Beams, H. W., Sedar, A. W. and Evans, T. C. 1953. La cellule 55: 291.

- Tahmisian, T. N., Devine, R. L. and Anderson, E. 1956. J. Biophys. biochem. Cytol. 2: 123.

Burgos, M. H. and Fawcett, D. W. 1956. J. Biophys. Biochem. Cytol. 1: 287.

Dalton, A. J. and Felix, M. D. 1954. Am. J. Anat. 94: 171.

- and - 1956. J. Biohys. Biochem. Cytol. 2: 79.

De Robertis, E. D. F. and Bennet, H. S. VIIIth Internat. Cong. Cell Biol., Leiden, 1954. Symp. on Fine Structure of Cells. Interscience Publishers, Inc. 1956: 261.

Gresson, R. A. R., Threadgold, L. T. and Stinson, N. E. 1956. La Cellule 58: 7.

Gatenby, J. E., Dalton, A. J. and Felix, M. D. 1955. Nature 176: 301.

Kitada, J. 1954. Bull. Naniwa Univ. 4: 121.

Lacy, D., and Rogers, G. E. 1955. J. Roy. Micr. Soc. 75: 172.

Moussa, T. A. A. 1950. J. Morph. 87: 27.

Malhotra, S. R. 1955. Quart. J. Micr. Sci. 97: 177.

Palay, S. L. and Palade, G. E. 1955. J. Biophys. Biochem. Cyto1. 1: 69.

Roque, A. L. 1954. J. Roy. Micr. Soc. 74: 188.

Sjöstrand, F. S. and Hanzon, V. 1954. Exp. Cell. Res. 7: 415.

Shafiq, S. A. 1953. Quart. J. Micr. Sci. 94: 319.

- 1954. Quart. J. Micr. Sci. 95: 305.

Thomas, O. L. 1947. Quart. J. Micr. Sci. 88: 445.

Watanabe, Y., Takamatsu, M. and Osako, R. 1956. Electron Microscopy, Tokyo 4: 145. 